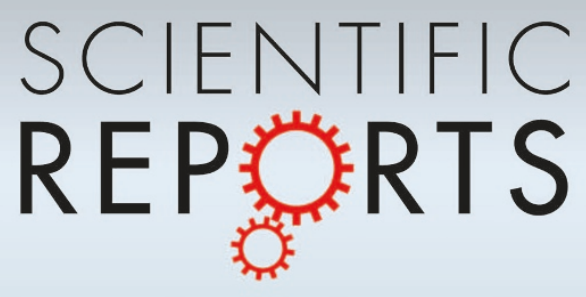

OPEN

SUBJECT AREAS:

ATOMISTIC MODELS

STRUCTURAL PROPERTIES

SURFACES, INTERFACES AND

THIN FILMS

METALS AND ALLOYS

Received

1 July 2013

Accepted

30 July 2013

Published

15 August 2013

Correspondence and requests for materials should be addressed to

J.W. (wangj6@lanl.

gov)

\section{Spiral Patterns of Dislocations at Nodes in (1 11 1) Semi-coherent FCC Interfaces}

\author{
Shuai Shao', Jian Wang' 'Amit Misra² \& Richard G. Hoagland'
}

${ }^{1}$ MST-8, Los Alamos National Laboratory, Los Alamos, NM 87545 USA, ${ }^{2}$ MPA-CINT, Los Alamos National Laboratory, Los Alamos, NM 87545 USA.

In semi-coherent interface, a superposed network of interface dislocations accommodates the attendant coherency strains in the adjacent crystals and their intersections (referred to as nodes) can act as sinks and sources for point defects because of the low formation energy. Nodes in $\{111\}$ semi-coherent interfaces are characterized with a spiral pattern (SP), wherein the line direction of each dislocation entering a node curves. The structure of SP nodes is able to switch between condensed and expanded by either reaction with point defects or mechanical deformation. Due to the switching of the node structures, point defect formation energies at nodes can be significantly reduced. Combining atomistic simulation and dislocation theory, these features are proven universal corresponding to the node density and the character of interface dislocations.

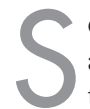
emi-coherent interfaces are key structural features in a wide range of engineering materials. Such interfaces arise, for example, in epitaxial layers, precipitation, and both diffusional and diffusionless displacive phase transformations ${ }^{1}$. A superposed network of interface dislocations accommodates the attendant coherency strains in the adjacent crystals ${ }^{2,3}$. Atomistic simulations combining in situ/ex situ transmission electron microscopy have provided insights into understanding the properties and their dependence on interface dislocation structures. It is found that interface dislocations may provide a diffusion pathway, facilitating the diffusion and reaction of point defects ${ }^{4-10}$. Interface dislocation intersections (referred to as nodes) may have low formation energy for point defects ${ }^{4,8,9}$. For instance, $\mathrm{Cu}(111) / \mathrm{Nb}(110)$ interface observed in layered composites was characterized by two sets of interface dislocations ${ }^{11-13}$. The low vacancy formation energy at nodes is accounted for by the expansion of jogs resulting from the reaction of interface dislocations ${ }^{11}$. Dislocation climb observed in in situ transmission electron microscopy ${ }^{14}$ and in atomistic simulations ${ }^{15}$ demonstrated high diffusivity for vacancies and interstitials along dislocation lines within interface. Examples of commonly observed $\{111\}$ semi-coherent interfaces in fcc metals include twist grain boundaries or twist/un-twist interphase boundaries. These boundaries have a low formation energy and hence, high thermal stability ${ }^{16-18}$. It seems well understood that such interfaces contain three sets of dislocations $(a / 2<110>$ or $a / 6<112>)$, depending on the stacking fault energy ${ }^{19-22}$. However, the node structure is rarely studied with respect to the node density and the character of interface dislocations. In this Letter, we studied $\mathrm{Cu}(111) / \mathrm{Ni}(111)$ semi-coherent interface by using molecular static/ dynamics (MS/MD) methods with empirical potential ${ }^{23}$ and characterized the interface by the disregistry analysis ${ }^{24}$. In recent years, many works have been carried out for $\mathrm{Cu}(111) / \mathrm{Ni}(111)$ interface and devoted to understand semi-coherent interface strengthening mechanisms in multilayers ${ }^{25-28}$. Here, we elucidate the atomic structure of the dislocation nodes in $\{111\}$ fcc interfaces and the response of such interface structures to point defects or mechanical shear. The details of atomistic simulations can be found in Method.

\section{Results}

Interface dislocation structure. (111) semi-coherent interface contains either three sets of Shockley partial dislocations $(a / 6<112>)$ or three sets of full dislocations $(a / 2<110>)$, depending on the stacking fault energy (as demonstrated in Fig. S2 in Supplemental materials). The most intriguing finding is that the dislocations in the vicinity of each node are reconfigured into a spiral pattern (SP), wherein the line direction of each dislocation entering a node curves in the same sense (Fig. 1(b)). With an un-relaxed bilayer as reference (Fig. 1(a)), the interface can be classified into four regions, near-fcc $\left(\mathrm{A}_{\mathrm{Ni}} \mathrm{B}_{\mathrm{Ni}} \mathrm{C}_{\mathrm{Cu}} \mathrm{A}_{\mathrm{Cu}}\right.$, normal (111) stacking between the $1^{\text {st }} \mathrm{Ni}$ and $1^{\text {st }} \mathrm{Cu}$ layers, $)$, near-hcp $\left(\mathrm{A}_{\mathrm{Ni}} \mathrm{B}_{\mathrm{Ni}} \mathrm{A}_{\mathrm{Cu}} \mathrm{B}_{\mathrm{Cu}}\right.$, the low-energy stacking fault between the $1^{\text {st }} \mathrm{Ni}$ and $1^{\text {st }} \mathrm{Cu}$ layers), the region separating the near-fcc from near-hcp regions, and the node region $\left(\mathrm{A}_{\mathrm{Ni}} \mathrm{B}_{\mathrm{Ni}} \mathrm{B}_{\mathrm{Cu}} \mathrm{C}_{\mathrm{Cu}}\right.$, high-energy 

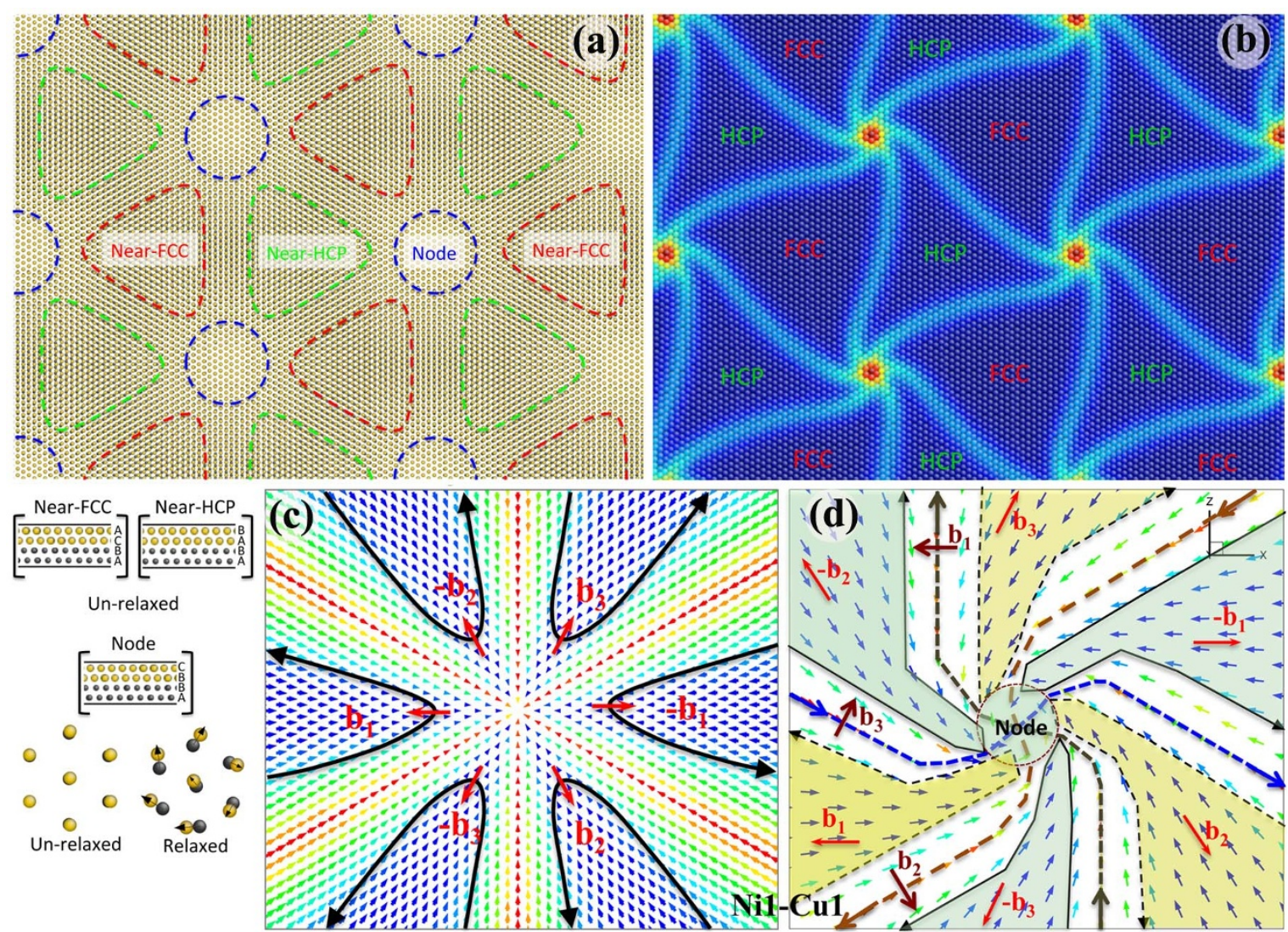

Figure 1 | Spiral patterns in $\mathrm{Cu}(111) / \mathrm{Ni}(111)$ interface. (a) Un-relaxed interface (yellow for Cu and black for Ni). (b) Relaxed interface (atoms are colored according to the excess potential energy). Interface dislocation lines align the light blue atoms. Disregistry analysis of (c) the un-relaxed interface and (d) the relaxed interface with respect to a reference fcc lattice (Ni) reveals formation mechanisms of interface dislocations. The arrows indicate the magnitude and direction of the disregistry. The bold-dashed lines in the color of dark brown, blue, and brown denote the resultant interface dislocations with Burgers vector $\boldsymbol{b}_{1}, \boldsymbol{b}_{3}$, and $\boldsymbol{b}_{2}$, respectively. Stacking fault regions are highlighted in yellow shadow and normal stacking regions are in cyan shadow.

stacking fault between the $1^{\text {st }} \mathrm{Ni}$ and $1^{\text {st }} \mathrm{Cu}$ layers). "Near" means that $\mathrm{Cu}$ and $\mathrm{Ni}$ crystals do not occupy same lattice sites due to the lattice mismatch in un-relaxed structures. Upon relaxation, the reduction in chemical potential energy drives the near-fcc and near-hcp regions to become fcc and hcp regions separated by Shockley partial dislocations. Fig. 1(c) and (d) show the disregistry around a node, indicating six Shockley partial dislocation loops. These dislocations have Burgers vectors, $\boldsymbol{b}_{1},-\boldsymbol{b}_{3}, \boldsymbol{b}_{2},-\boldsymbol{b}_{1}, \boldsymbol{b}_{3}$, and $-\boldsymbol{b}_{2}$. Where $\boldsymbol{b}_{1}=\frac{a}{6}[2 \overline{1} \overline{1}]$, $\boldsymbol{b}_{2}=\frac{a}{6}[\overline{1} 2 \overline{1}]$, and $\boldsymbol{b}_{3}=\frac{a}{6}[\overline{1} \overline{1} 2]$. The reaction between the adjacent loops results in three sets of edge dislocations with Burgers vector of $\boldsymbol{b}_{1}, \boldsymbol{b}_{2}$, and $\boldsymbol{b}_{3}$ (Fig. 1(d)). At the node region, the high-energy stacking fault can only be relaxed through the twist between $\mathrm{Cu}$ and $\mathrm{Ni}$ because of the three-fold symmetric structure. Atoms near the center of the node can only rotate around the center, and occupy either fcc or hcp sites. Both lattice sites have lower energy than the high-energy stacking fault sites. Moreover, this twist causes the curved interface dislocation lines, changing the character of interface dislocations near the node from an edge-type dislocation to a mixed-type or even a screw-type dislocation. Corresponding to elastic theory of dislocation, both types of dislocations have lower self-energy than an edge-type dislocation. Thus, the SP associated with changes in dislocation character and the high-energy stacking fault is energetically and kinetically favored.

Expanded node under mechanical shearing. Under the applied shear stress along the $\langle 11 \overline{2}\rangle$, MD simulations revealed the expanded node as shown in Fig. 2(a). The node spreads itself and forms an extrinsic stacking fault. Differing from the condensed node, dislocations at the expanded node are lying on two planes (Fig. 2(b)). One set of dislocations is lying in Nil-Cul interface and the other in the $\mathrm{Cu} 1-\mathrm{Cu} 2$ plane. Dislocation jogs form and bridge these dislocations in the two planes. Corresponding to the new dislocation structure at the expanded node, Fig. 2(c) and (d) show the change in atomic structures of the first $\mathrm{Cu}$ layer. This is consistent with the observation that the termini of the extrinsic regions contain jogs and the expansion of the extrinsic area requires climb at the jogs by diffusion ${ }^{29}$.

The disregistry analysis provides insight into understanding the expanded node. On the Nil-Cul interface, the dashed-line dislocation loops (enclosing stacking fault highlighted in yellow shadow) expand towards the center of the node, while the solid-line dislocation loops (enclosing normal stacking highlighted in cyan shadow) retract (Fig. 3(a) and (b)). The dashed-line loops then react and form three pure screw dislocations (red dashed lines) around the node (Fig. 3(b) and (d)). On the Cu1-Cu2 plane, three partial dislocation loops form in association with the motion of dislocations on Ni1-Cu1 plane, react and result in three pure screw dislocations and three segmental partial dislocations (Fig. 3(c) and (e)). The screw dislocations on Ni1-Cu1 and $\mathrm{Cu} 1-\mathrm{Cu} 2$ planes have the same Burgers vectors $\boldsymbol{b}_{4}, \boldsymbol{b}_{5}$, and $\boldsymbol{b}_{6}$ but the opposite line senses, respectively. Hence they annihilate. Here $\boldsymbol{b}_{4}=\frac{a}{2}[1 \overline{1} 0], \boldsymbol{b}_{5}=\frac{a}{2}[\overline{1} 01], \boldsymbol{b}_{5}=\frac{a}{2}[01 \overline{1}]$. The resultant dislocation structures at a node are schematically shown in Fig. 2(b). The change in node structure only occurs in the $\mathrm{Cu}$ side because the free volume in association with the tension on $\mathrm{Cu}$ side at nodes can be delocalized in three newly formed dislocation jogs (Fig. 2(d)). 

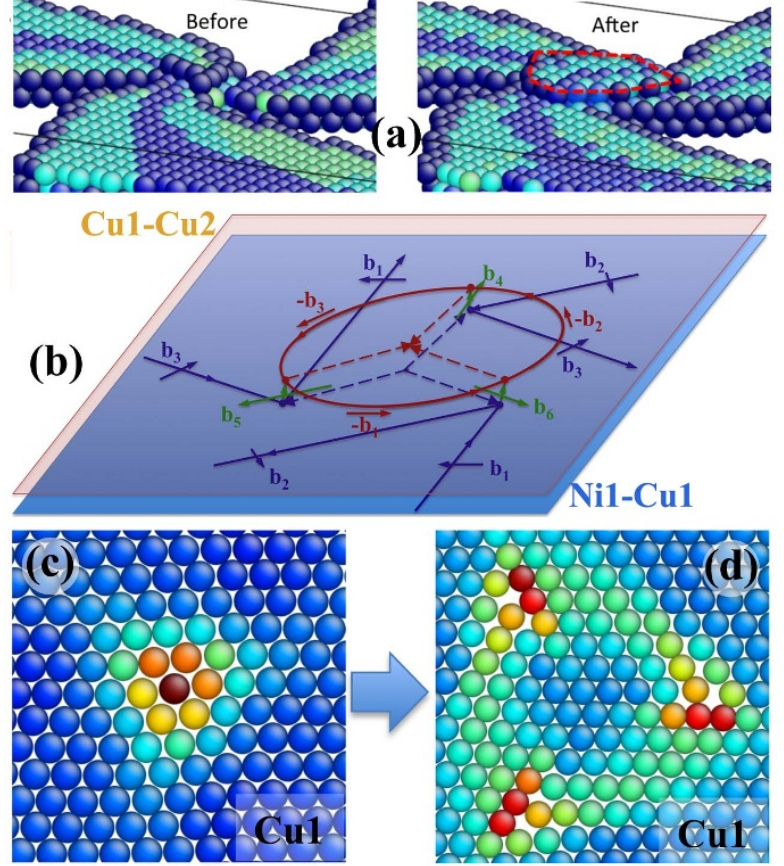

Figure $2 \mid$ The change in the node structure under mechanical shear parallel to interface. (a) Atomic structures of the condensed and expanded nodes. The red dashed circle outlines the expanded region. (b) Dislocation structure at the expanded node. The blue dislocation lines lie in the Ni1-Cu1 interface, the brown lines in the $\mathrm{Cu} 1-\mathrm{Cu} 2$ interface, and the green lines indicate dislocation jogs. $\mathrm{Cu} 1$ and $\mathrm{Nil}$ are the first $\mathrm{Cu}$ and $\mathrm{Ni}$ atomic layers from the interface, respectively. The change in atomic structure of the first $\mathrm{Cu}$ layer from the condensed node (c) to the expanded node (d). Atoms are colored according to the excess potential energy.
Vacancy formation at nodes. For a condensed node, vacancy formation energy (VFE) at the dislocation cores is $1.28 \mathrm{eV}$ on $\mathrm{Cu}$ side (close to $1.29 \mathrm{eV}$ in bulk) and $1.2 \mathrm{eV}$ on $\mathrm{Ni}$ side (less than $1.51 \mathrm{eV}$ in bulk). This is expected because vacancy formation on the $\mathrm{Cu}$ side is unfavorable due to the hydrostatic tension at nodes. For both condensed and expanded nodes, VFE within fcc and hcp regions is close to that in bulk, $1.23 \mathrm{eV}$ on $\mathrm{Cu}$ side and $1.44 \mathrm{eV}$ on Ni side. But VFE at nodes is significantly lower than that in bulk $\mathrm{Cu}$ and Ni.

At nodes, VFE on Ni side is around $0.54 \mathrm{eV}$ (Fig. 4(a)) for the condensed node and $0.04 \mathrm{eV}$ for the expanded node (Fig. 4(b)). Both are significantly lower than the bulk VFE in $\mathrm{Ni}(1.51 \mathrm{eV})$. It is interesting that VFE on the $\mathrm{Cu}$ side has also dropped to $0.95 \mathrm{eV}$ from $1.29 \mathrm{eV}$ in the bulk although the region is under hydrostatic tension (Fig. 4(c)). The analysis of atomic structure at nodes reveals that a $\mathrm{Ni}$ atom fills in the vacancy site where a $\mathrm{Cu}$ atom is removed. Consequently, the elastic strain energy on the $\mathrm{Ni}$ side at the node will decrease. For the expanded node, VFE on the $\mathrm{Cu}$ side is $0.67 \mathrm{eV}$ when the vacancy is created close to a jog in the Cul layer (Fig. 4(d)). The lower formation energy is associated with the climb of the jog by absorbing the vacancy (Fig. 4(e)). When a vacancy in Cul layer is created in the center of the extrinsic stacking fault, a $\mathrm{Ni}$ atom fills in the vacancy site, and VFE is about $0.47 \mathrm{eV}$. Interestingly, the expanded node collapses into a condensed node (Fig. 4(f)). When a vacancy is created on the $\mathrm{Ni}$ side, a $\mathrm{Cu}$ atom also fills in the vacancy site (Fig. 4(g)), consequently the expanded node condenses and VFE is close to $0.05 \mathrm{eV}$. Compared to the condensed node, a quite significant decrease of VFE in the expanded node is always observed, from $0.95 \mathrm{eV}$ to $0.47 \mathrm{eV}$ in $\mathrm{Cu}$ and from $0.54 \mathrm{eV}$ to $0.05 \mathrm{eV}$ in $\mathrm{Ni}$, while a node condensation process takes place. This can be attributed to the facts that the expanded node has a slightly high energy and the $\mathrm{Cu}$ vacancy facilitates jog motion and an overall lowering of dislocation interaction energy.

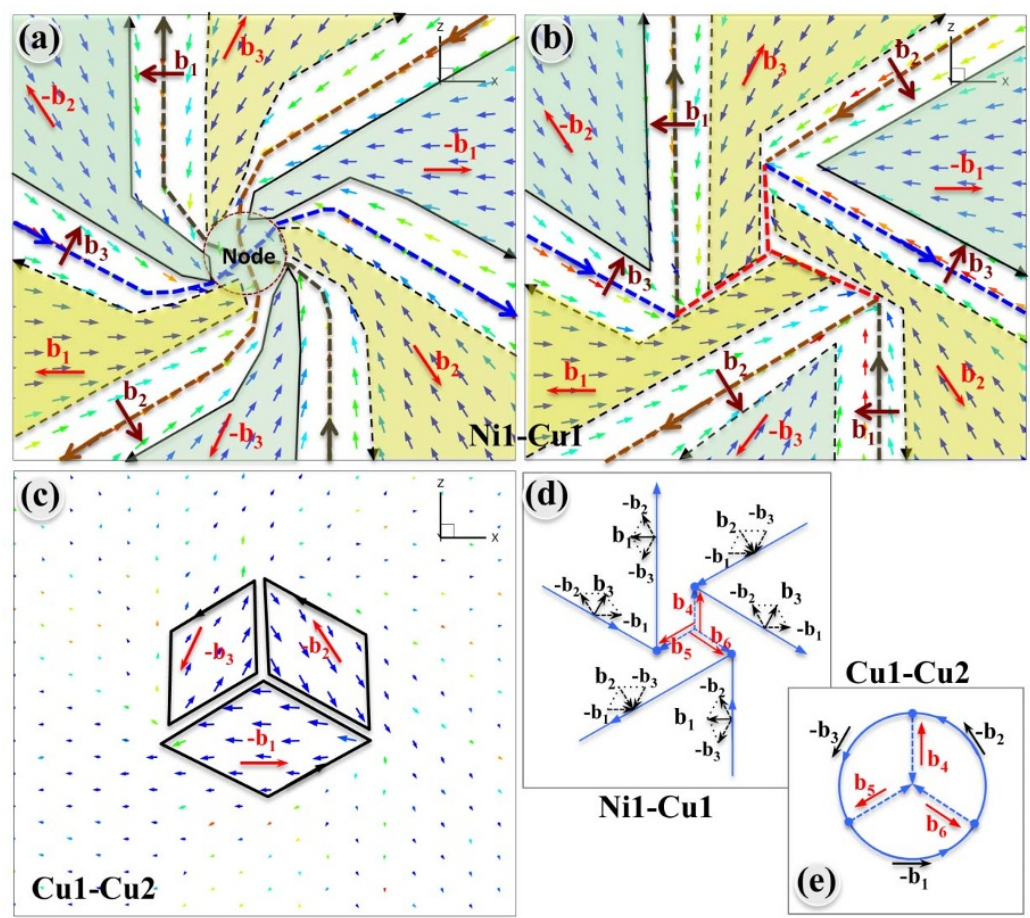

Figure 3 Dislocation structures at the expanded node. (a) and (b) disregistry in Nil-Cul plane (a) before and (b) after the node expansion. (c) Disregistry in Cu1-Cu2 plane after the node expansion. The bold dashed lines indicate the interface dislocations. The yellow regions are hcp stacking and the cyan regions are normal fcc stacking. Dislocation structures, (d) on Ni1-Cu1 plane and (e) on Cu1-Cu2 plane, after the node expansion. Dashedline dislocations on the two planes (d) and (e) with Burgers vectors, $\boldsymbol{b}_{4}, \boldsymbol{b}_{5}$, and $\boldsymbol{b}_{6}$ annihilate, and are not presented in the node region. 

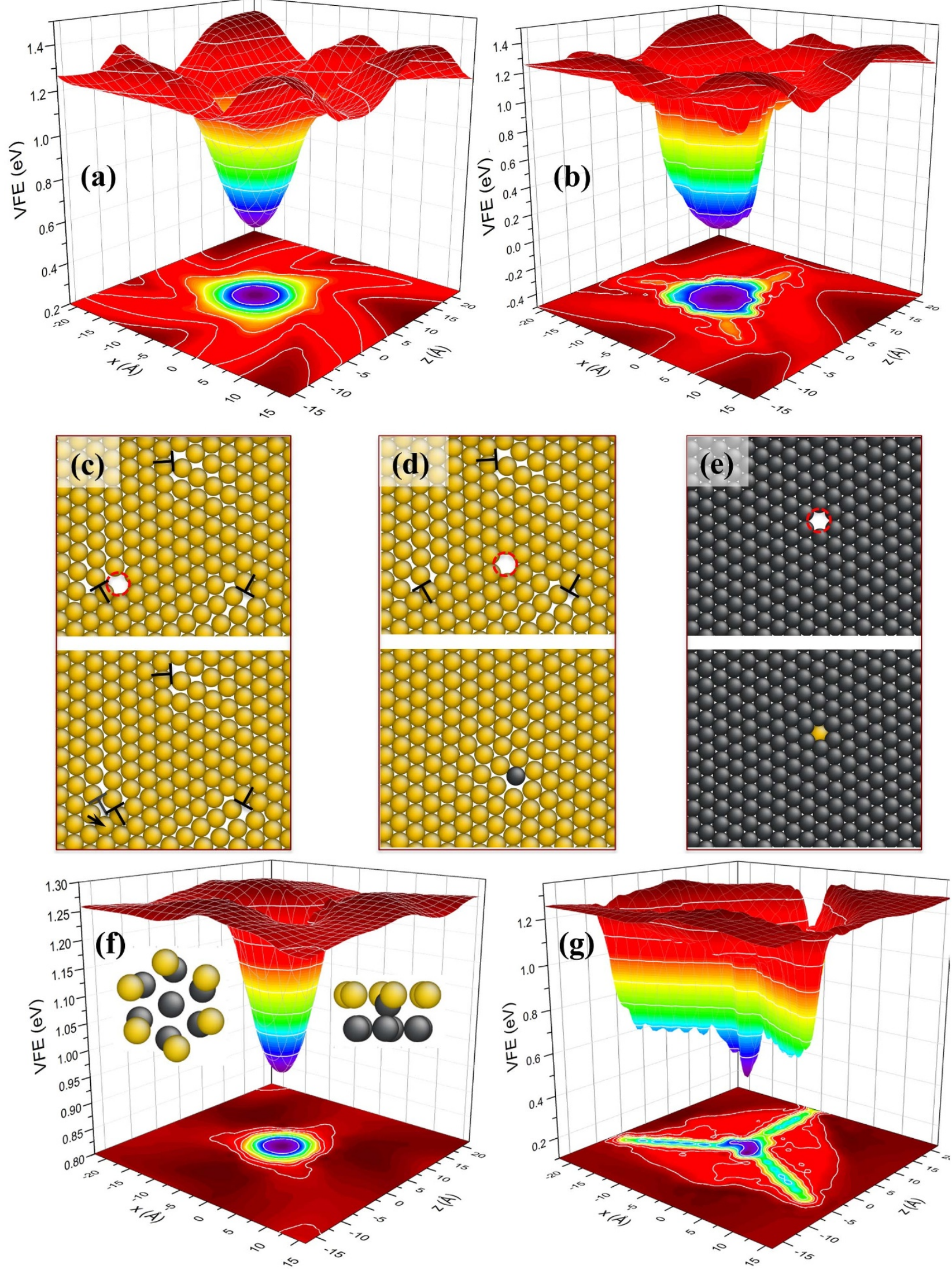

Figure $4 \mid$ Structures and energies of vacancy at nodes. Energy contours of vacancy formation energy in Ni side (a) for the condensed node and (b) for the expanded node. Changes in atomic structures, (c) the jog climbs after a vacancy created in Cul layer near the jog, (d) a Ni atom fills in the vacancy site created in Cul layer away from the jogs, and (e) a Cu atom fills in the vacancy site created in Ni1 layer. Cu atoms are colored in the orange and $\mathrm{Ni}$ atoms in black. The vacancy sites are denoted in a red circle. Energy contours of vacancy formation energy in Cu for (f) the condensed node and (g) the expanded node. The lowest formation energy corresponds to the $\mathrm{Ni}$ atom (black) motion towards the vacancy site in $\mathrm{Cu}$ (yellow). 

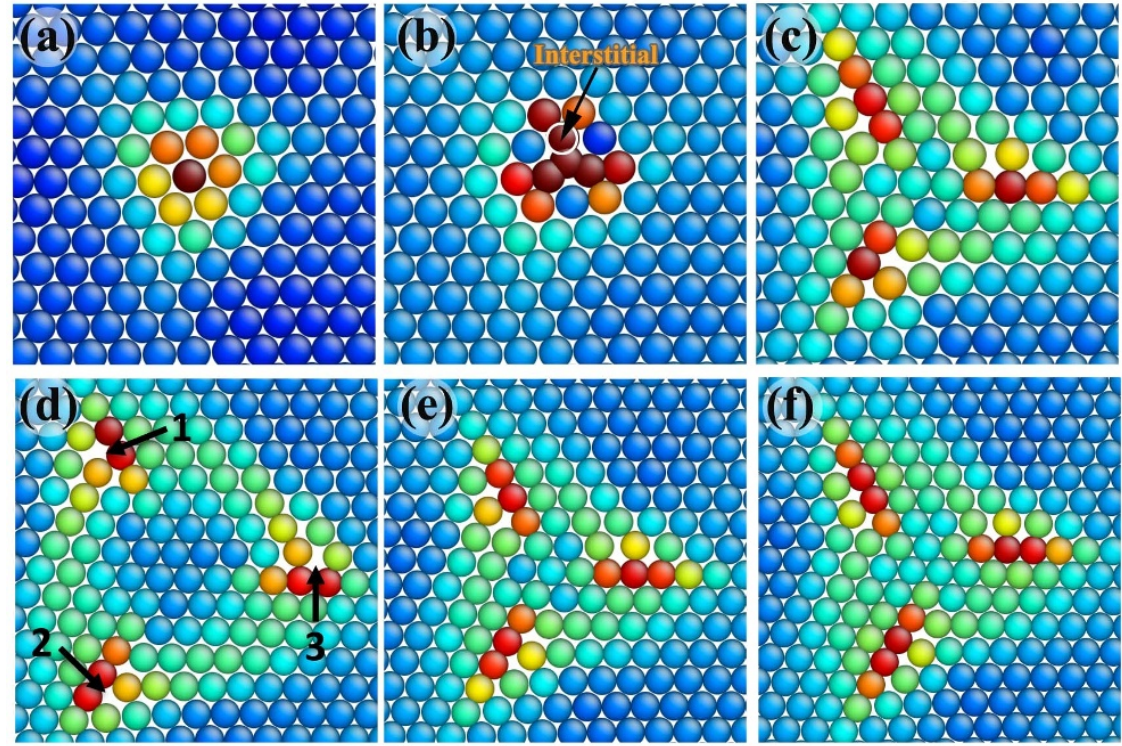

Figure $5 \mid$ Atomic structures of Cul layer for the condensed node. (a) before inserting a Cu interstitial, (b) insertion without relaxation, and (c) after relaxation, respectively. Atomic structures of Cul layer for the expanded node: (d) before inserting a Cu interstitial, (e) after relaxation with one interstitial in dislocation jog (1), and (f) after relaxation with three interstitials in three dislocation jogs, respectively. Atoms are colored according to the excess potential energy.

Interstitial formation at nodes. Motivated by the change in node structures accompanying the reaction of node with vacancy in $\mathrm{Ni}$, $\mathrm{MD}$ simulations demonstrated that a $\mathrm{Cu}$ interstitial also results in the expanded node (Fig. 5(a)-(c)). Self-interstitial formation energy (SIFE) is computed to be $-0.14 \mathrm{eV}$ when a $\mathrm{Cu}$ atom is embedded into the center of the node. For the expanded node (Fig. 5(d)), SIFE is around $-0.65 \mathrm{eV}$ when a $\mathrm{Cu}$ atom is embedded into one of three dislocation jogs (Fig. 5(e)). When three $\mathrm{Cu}$ atoms are simultaneously embedded into the three jogs (Fig. 5(f)), the average SIFE is $-0.62 \mathrm{eV}$. The expanded node shrinks accompanying the absorption of $\mathrm{Cu}$ interstitials. The diameter of the expanded node decreases from $2.2 \mathrm{~nm}$ to $1.4 \mathrm{~nm}$. The results imply that the expanded node can be a stronger sink for interstitials, as compared with SIFE in the condensed node, and bulk $\mathrm{Cu}(3.08 \mathrm{eV})$ and $\mathrm{Ni}(4.64 \mathrm{eV})$. In addition, SIFE in the $\mathrm{fcc}$ and hcp regions is $3.7 \sim 3.9 \mathrm{eV} /$ atom in-between that in bulk $\mathrm{Cu}$ and $\mathrm{Ni}$ with a $<100>$-dumbbell configuration that is similar to that in fcc crystals ${ }^{30}$.

\section{Discussion}

Are these observed features universal in fcc semi-coherent interface? We examined the universality of both the node expansion and the SP feature with respect to two types of interfaces. One is twist boundary in fcc single crystals ( $\mathrm{Ag}, \mathrm{Cu}$, and $\mathrm{Al}$ ), and the other is the $\mathrm{Cu}-\mathrm{Ag}$ system with a large lattice mismatch of $13 \%$. MD simulations show that both features disappear in the twist boundaries and the $\mathrm{Cu}-\mathrm{Ag}$ interface (Figs. S3 and S4 in Supplemental materials). It is understandable for twist boundaries, because (1) interface dislocations are all pure screw-type Shockley partials, (2) a node is not subject to zero tension and compression due to zero lattice mismatch, and (3) atoms in the node region before relaxation have already occupied lowenergy stacking faulted sites. This result suggests the strong dependence of node structure on the character of interface dislocations. The disappearance of the SP feature in $\mathrm{Cu}$-Ag system could be ascribed to the competition among self-energies of the nodes and interface dislocations and the interaction energy among them because of the shorter distance between the adjacent nodes $(\sim 2.2 \mathrm{~nm}$ in $\mathrm{Cu}-\mathrm{Ag}$ and $\sim 9.5 \mathrm{~nm}$ in $\mathrm{Cu}-\mathrm{Ni}$ system). Moreover, the distance between nodes, $2.2 \mathrm{~nm}$, is close to the diameter of an expanded node. If a node expands in association with the creation of three jogs, the significantly high repulsion between jogs in association with the adjacent nodes due to the non-linear interaction ${ }^{22}$ will suppress such kind of expansion. The result implies the dependence on the distance between nodes (corresponding to lattice mismatch and the twist angle).

Figure 6 summarizes the dependence of the SP feature at nodes on the character of interface dislocations and the distance between the adjacent nodes. The distance between nodes can be calculated according to Frank-Bilby theory ${ }^{2,3}$. The critical distance corresponding to the disappearance of the SP feature is $2.2 \mathrm{~nm}$ according to the expanded node in $\mathrm{Cu} / \mathrm{Ni}$ interface. With respect to the character of interface dislocations, (111) semi-coherent interfaces in fcc crystals can be further categorized into (i) pure twist boundary in single phase $\left(b_{\mathrm{e}} / b=0\right.$, referred to as No Mismatch \& Pure Twist), (ii) bi-crystal boundary in the same orientations containing $\left(b_{\mathrm{e}} / b=1\right.$, referred to as Mismatch \& No Twist), and (iii) mixed boundary $\left(0<b_{\mathrm{e}} / b<1\right.$, referred to as Mismatch \& Twist). For type 1, nodes don't twist and expand. For type 2 interfaces with the larger lattice mismatch (such as $\mathrm{Cu} / \mathrm{Ag}$ ), nodes would not twist and expand. Thus, an optimized (111) semi-coherent interface (Type 3 interfaces) with respect to sink strength for point defects should exist for a given system. For example, $\mathrm{Cu}-\mathrm{Ni}$ interface can be further twisted to increase the node density while retaining the SP feature at nodes (Fig. 6).

It is worth pointing out that interface dislocations can act as sources for nucleation and emission of lattice dislocation into the adjacent layers during mechanical deformation ${ }^{31,32}$. Atomistic scale models are able to provide insight into characterizing the characteristic of interface dislocations and their nodes, but not able to predict macroscopic properties of nanolayered composites. Dislocation Dynamics models have the unique advantage of exploring the evolution of interface dislocation network and the activity of interface dislocations during mechanical deformation at large scale ${ }^{33,34}$, but the dislocation reaction rules related to interface properties have to be implemented according to atomistic simulations. For example, the screw dislocation segments at the nodes in association with the expanded structure could be a source for emission of lattice dislocation into the adjacent layer through cross-slip mechanism. Thus a predictive materials modeling tool by advancing the Dislocation Dynamics method and coupling with atomistic-level deformation 


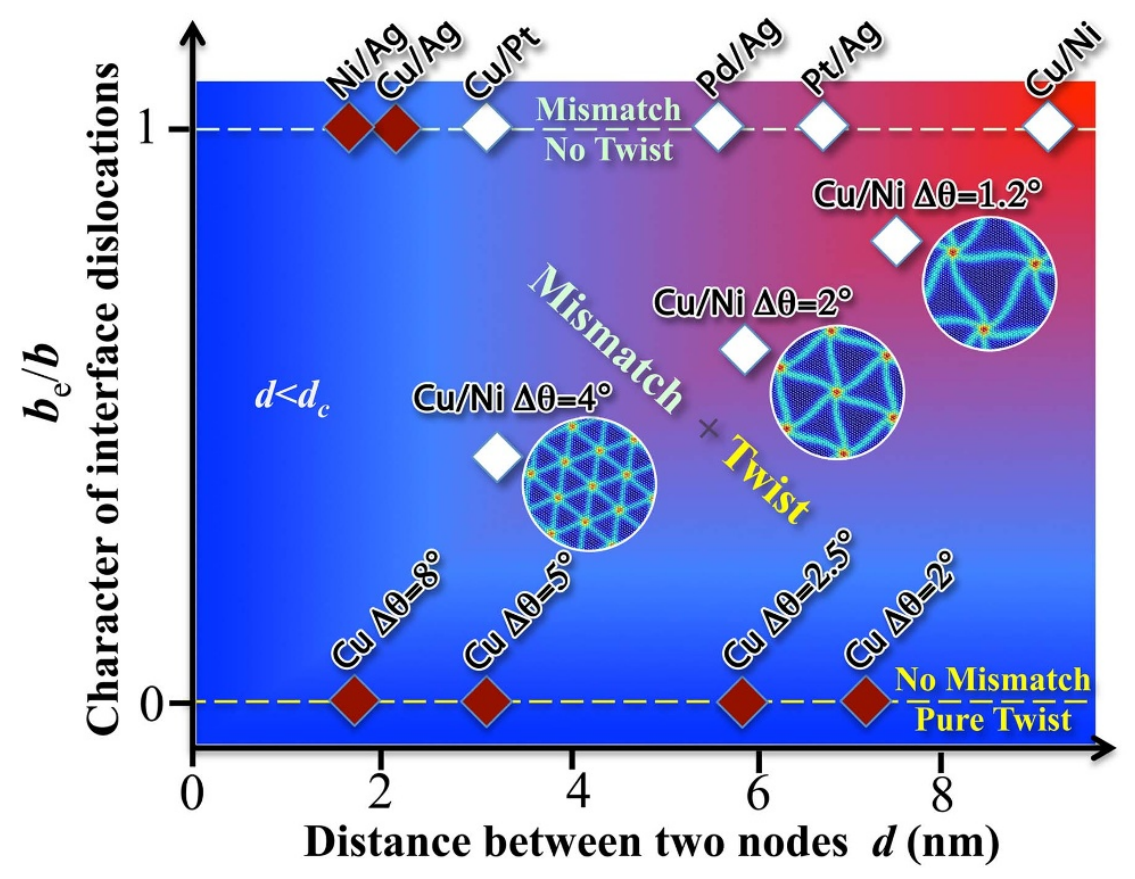

Figure $6 \mid$ Dependence of the SP feature at nodes on the distance between two nodes and the character of interface dislocations. $\boldsymbol{b}_{\mathrm{e}}$ is the edge component of Burgers vector $\boldsymbol{b}$. The white diamonds indicate the presence of the SP feature in the interface. The brown diamonds indicate the disappearance of the SP feature in the interface. The red region indicates the SP feature in association with both the condensed and the expanded nodes. Blue region indicates the condensed node without the SP feature.

mechanisms within/at/across boundaries would be promising to bridge the length-scale gap from atomic-scale to meso/macro-scale.

In summary, the nodes in (111) semi-coherent interface in fcc crystals can adopt the condensed or the expanded structures with/ without the SP feature. The change in node structure can be achieved via either mechanical shearing or interstitial absorption. When the interface is unstable chemically, various types of nodes may cause chemical mixing and destruct interface structure. However, this does not occur when they are chemically immiscible. The significant decrease of formation energy of vacancy and interstitial in association with the structure change of nodes makes such kind of interface as superior sinks for point defects that are created by either severe plastic deformation or irradiation. More importantly, these structural features are correlated with lattice mismatch and the character of interface dislocations.

\section{Methods}

$\mathrm{Cu}(111) / \mathrm{Ni}(111)$ semi-coherent interface is chosen in this study. Molecular static/ dynamics (MS/MD) simulations are conducted with the embedded atom method interatomic potentials for $\mathrm{Cu}^{35}$ and $\mathrm{Ni}^{36}$, and their cross-pair ${ }^{23}$. The two crystals have the same coordinate, $x$ along $[11 \overline{2}], y$ along [111], and $z$ along [1ํㅣㅇ. The interface plane is in the $x$ - $z$ plane. The periodic boundary conditions are applied for the $x$ and $z$ directions. The fixed boundary condition is applied for the $y$ direction. Fig. S1 shows simulation cell in Supplementary materials. Structures of interface dislocations and nodes are analyzed and characterized by the disregistry analysis. Interface dislocation structures (including dislocations and nodes) are further understood by using dislocation models. The formation energies of point defects (vacancy and self interstitial) are calculated using the MS/MD methods. After creating a point defect, the system is relaxed at finite temperature of $10 \mathrm{~K}$ for $20 \mathrm{ps}$ and followed by quenching-MD until the maximum force on atoms is less than $5 \mathrm{pN}$. By applying mechanical shear stresses parallel to interface plane or inserting additional atoms in the nodes, we observed the change in the node structure, and accounted for the change based on the creation and reaction of dislocations. Accompanying the atomic rearrangements at the nodes, we also calculated the formation energies of a vacancy and a self-interstitial. Node expansion under mechanical shearing is simulated by applied a constant shear strain rate $\left(\dot{\varepsilon}_{y x}=5 \times 10^{8} / \mathrm{s}\right)$ on the bilayer parallel to the interface ${ }^{24}$. A finite temperature (T $=1 \mathrm{~K})$ is maintained in the system through out the loading process. The first node expansion is observed at a fairly small strain $\left(\varepsilon_{y x}=0.01\right)$. In the computational cell there exists only two nodes. Therefore, due to periodicity, half of the node population of the entire interface has expanded. The expansion of the rest of the nodes happens at $\varepsilon_{y x}=0.03$. The misfit dislocation network also migrates to accommodate the imposed strain. The expansion is a thermally activated process and the fact that it takes place at small strains suggests that the energy barrier between two states (condensed and expanded) of the node is relatively small. After the node expansion, the inverse loading was applied until the system is equilibrated under zero stresses. Dislocation structures and atomic structures at nodes are analyzed for the relaxed, equilibrium interface at zero temperature. The interface formation energy of the expanded node is $0.324 \mathrm{~J} / \mathrm{m}^{2}$, slightly higher than that of the condensed node $\left(0.322 \mathrm{~J} / \mathrm{m}^{2}\right)$. The difference mainly arises from the increase of the node energy by about $0.5 \mathrm{eV}$.

1. Howe, J. M., Pond, R. C. \& Hirth, J. P. The role of disconnections in phase transformations. Prog. Mater. Sci. 54, 792-838 (2009).

2. Hirth, J. P., Pond, R. C., Hoagland, R. G., Liu, X. Y. \& Wang, J. Interface defects, reference spaces and the Frank-Bilby equation. Prog. Mater. Sci. 58, 749-823 (2013).

3. Wang, J. et al. Characterizing interface dislocations by atomically informed Frank-Bilby theory. J. Mater. Res. 28, 1646-1657 (2013).

4. Di, Z. F. et al. Tunable helium bubble superlattice ordered by screw dislocation network. Phys. Rev. B 84, 052101 (2011).

5. Li, N. et al. A. Incoherent twin boundary migration induced by ion irradiation in Cu. J. Appl. Phys. 113, 023508 (2013).

6. Rose, M., Balogh, A. G. \& Hahn, H. Instability of irradiation induced defects in nanostructured materials. Nuc. Instr. Meth. Phys. Res. B 127/128, 119-122 (1997).

7. Chimi, Y. et al. Accumulation and recovery of defects in ion-irradiated nanocrystalline gold. J. Nuc. Mater. 297, 355-357 (2001).

8. Martínez, E. \& Caro, A. Atomistic modeling of long-term evolution of twist boundaries under vacancy supersaturation. Phys. Rev. B 86, 214109 (2012).

9. Martínez, E., Hirth, J. P., Nastasi, M. \& Caro, A. Structure of a 2 degrees (010) Cu twist boundary interface and the segregation of vacancies and He atoms. Phys. Rev. B 85, 060101(R) (2012).

10. Kolluri, K. \& Demkowicz, M. J. Formation, migration, and clustering of delocalized vacancies and interstitials at a solid-state semicoherent interface. Phys. Rev. B 85, 205416 (2012).

11. Demkowicz, M. J., Hoagland, R. G. \& Hirth, J. P. Interface structure and radiation damage resistance in $\mathrm{Cu}-\mathrm{Nb}$ multilayer nanocomposites. Phys. Rev. Lett. 100, 136102 (2008).

12. Demkowicz, M. J., Wang, J. \& Hoagland, R. G. in Dislocations in Solids (Ed. Hirth, J. P.). Interfaces Between Dissimilar Crystalline Solids, Vol. 14, Chap. 83, p. 141-207 (Elsevier, Amsterdam, 2008).

13. Wang, J. et al. Interface Dislocation Patterns and Dislocation Nucleation in Facecentered-cubic and Body-centered-cubic Bi-crystal Interfaces. Int. J. Plasticity. http://dx.doi.org/10.1016/j.ijplas.2013.07.002. 
14. Li, N., Wang, J., Huang, J. Y., Misra, A. \& Zhang, X. In situ TEM observations of room temperature dislocation climb at interfaces in nanolayered $\mathrm{Al} / \mathrm{Nb}$ composites. Script. Mater. 63, 363-366 (2010).

15. Wang, J., Hoagland, R. G. \& Misra, A. Room-temperature dislocation climb in metallic interfaces. App. Phys. Lett. 94, 131910 (2009)

16. Wang, J., Beyerlein, I. J., Mara, N. A. \& Bhattacharyya, D. Interface-facilitated deformation twinning in copper within submicron Ag-Cu multilayered composites. Script. Mater. 64, 1083-1086 (2011).

17. Tian, Y.Z. \& Zhang, Z. F. Stability of interfaces in a multilayered Ag-Cu composite during cold rolling. Script. Mater. 68, 542-545 (2013).

18. Lauwaet, K., Schouteden, K., Janssens, E., Van Haesendonck, C. \& Lievens, P. Dependence of the $\mathrm{NaCl} / \mathrm{Au}(111)$ interface state on the thickness of the $\mathrm{NaCl}$ layer. J. Phys.: Condens. Matter. 24, 475507 (2012).

19. Cheng, D., Yan, Z. J. \& Yan, L. Misfit dislocation network in $\mathrm{Cu} / \mathrm{Ni}$ multilayers and its behaviors during scratching. Thin Solid Films 515, 3698-3703 (2007).

20. Wu, W. P., Guo, Y. F., Wang, Y. S., Mueller, R. \& Gross, D. Molecular dynamics simulation of the structural evolution of misfit dislocation networks at gamma/ gamma phase interfaces in Ni-based superalloys. Phil. Mag. 91, 357-372 (2011).

21. Zepeda-Ruiz, L. A., Maroudas, D. \& Weinberg, W. H. Semicoherent interface formation and structure in InAs/GaAs(111)A heteroepitaxy. Surf. Sci. 418, L68-L72 (1998).

22. Hirth, J. P. \& Lothe, J. Theory of Dislocaitons, $2^{\text {nd }}$ Edition. (Krieger Publishing Company, Malabar, FL 1992).

23. Bonny, G., Pasianot, R. C., Castin, N. \& Malerba, L. Ternary Fe-Cu-Ni many-body potential to model reactor pressure vessel steels: First validation by simulated thermal annealing. Phil. Mag. 89, 3531-3546 (2009).

24. Wang, J., Hoagland, R. G., Hirth, J. P. \& Misra, A. Atomistic simulations of the shear strength and sliding mechanisms of copper-niobium interface. Acta Mater. 56, 3109-3119 (2008).

25. Rao, S. I. \& Hazzledine, P. M. Atomistic simulations of dislocation-interface interactions in the Cu-Ni multilayer system. Phil. Mag. A 80, 2011-2040 (2000).

26. Hoagland, R. G., Mitchell, T. E., Hirth, J. P. \& Kung, H. On the strengthening effects of interfaces in multilayer fee metallic composites. Phil. Mag. A 82, 643-664 (2002).

27. Liu, Y., Bufford, D., Wang, H., Sun, C. \& Zhang, X. Mechanical properties of highly textured Cu/Ni multilayers. Acta. Mater. 59, 1924-1933 (2011).

28. Mitlin, D. et al. Formation of Misfit Dislocations in Nano-Scale Ni-Cu Bilayer Films. Phil. Mag. A 84, 719-736 (2004).

29. Carter, C. B. The influence of jogs on the extension of dislocation nodes. Phil. Mag A 41, 619-635 (1980).

30. Schilling, W. Self-interstitial atoms in metals. J. Nuc. Mater. 69-70, 465-489 (1978)

31. Zhang, R. F., Wang, J., Beyerlein, I. J., Misra, A. \& Germann, T. C. Atomic-scale study of nucleation of dislocations from fcc-bcc interfaces. Acta Mater 60, 2855-2865 (2012)
32. Zhang, R. F., Wang, J., Beyerlein, I. J. \& Germann, T. C. Dislocation nucleation mechanisms from fcc/bcc incoherent interfaces. Scripta Mater 65, 1022-1025 (2011).

33. Hughes, D. A., Khan, S. M. A., Godfrey, A. \& Zbib, H. M. Internal Structures of Deformation Induced Planar Dislocation Boundaries. Mater. Sci. and Eng. A 309-310, 202-226 (2001).

34. Khan, S., Zbib, H. M. \& Hughes, D. Modeling Planar Dislocation Boundaries Using a Multi-Scale Approach. Int. J. Plasticity. 20, 1059-1092 (2004).

35. Mishin, Y. et al. Structural stability and lattice defects in copper: Ab initio, tightbinding, and embedded-atom calculations. Phys. Rev. B 63, 224106 (2001)

36. Voter, A. F. \& Chen, S. P. Accurate Interatomic Potentials for Ni, Al and Ni3Al. Mater. Res. Soc. Symp. Proc. 82, 175-180 (1987).

\section{Acknowledgments}

This work was supported by the Center for Materials at Irradiation and Mechanical Extremes, an Energy Frontier Research Center funded by the U.S. Department of Energy, Office of Science, Office of Basic Energy Sciences under Grant No. 2008LANL1026. JW also thank the support provided by a Los Alamos National Laboratory Directed Research and Development project ER20140450. Authors are grateful for the helpful comments from Prof. John P. Hirth.

\section{Author contributions}

S.S. performed all MD simulations and wrote the first draft of the manuscript. J.W. designed this project and coordinated the discussion with A.M. and R.G.H. All authors finalized the manuscript.

\section{Additional information}

Supplementary information accompanies this paper at http://www.nature.com/ scientificreports

Competing financial interests: The authors declare no competing financial interests.

How to cite this article: Shao, S., Wang, J., Misra, A. \& Hoagland, R.G. Spiral Patterns of Dislocations at Nodes in (111) Semi-coherent FCC Interfaces. Sci. Rep. 3, 2448; DOI:10.1038/srep02448 (2013).

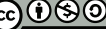

This work is licensed under a Creative Commons Attribution-

NonCommercial-ShareAlike 3.0 Unported license. To view a copy of this license, visit http://creativecommons.org/licenses/by-nc-sa/3.0 